\title{
ON SINGULAR PERTURBATION OF THE STOKES PROBLEM
}

\author{
G. M. KOBEL'KOV \\ Department of Mechanics and Mathematics, Moscow University \\ 119899 Moscow, Russia
}

In papers $[1,2]$ the perturbation of the Stokes problem was studied. We mean the case when the original Stokes problem for incompressible media is replaced by elasticity theory equations with Poisson ratio $\nu$ approximately equal to $1 / 2$. In this case it was proved that $u_{\varepsilon} \rightarrow u_{0}, p_{\varepsilon} \rightarrow p_{0}$ where $\varepsilon \sim 1 / 2-\nu,\left(u_{\varepsilon}, p_{\varepsilon}\right)$ is a solution of the boundary value problem for elasticity theory equations and $\left(u_{0}, p_{0}\right)$ is the solution of the Stokes problem. But in the papers mentioned above only the case $\varepsilon=$ const was considered. We will consider the case $\varepsilon=\varepsilon(x)$. Our technique is different but the results are almost the same. So let us consider the boundary value problem for elasticity theory equations when the Lamé coefficient $\mu$ is constant.

For the sake of simplicity of presentation we will consider the Dirichlet boundary conditions

$$
\begin{aligned}
\mu \Delta u+\nabla(\lambda+\mu) \operatorname{div} u & =F, \\
\left.u\right|_{\partial \Omega} & =0 .
\end{aligned}
$$

Unless otherwise stated, we will assume that $\mu=$ const. In this case, similarly to [3], the boundary value problem (1) can be rewritten in the following form:

$$
\begin{gathered}
-\Delta u+\nabla p=f, \\
\alpha p+\operatorname{div} u=0,\left.\quad u\right|_{\partial \Omega}=0 ;
\end{gathered}
$$

here $f=-F / \mu$ and $\alpha=\mu /(\lambda+\mu)$. It can be easily seen that the boundary value problem (2) has a more general form than (1) since it covers the case $\alpha=0$ on part of or on the whole domain $\Omega$.

1991 Mathematics Subject Classification: Primary 35Q30.

The paper is in final form and no version of it will be published elsewhere. 
Along with (2) let us consider the perturbed boundary value problem

$$
\begin{gathered}
-\Delta u_{\varepsilon}+\nabla p_{\varepsilon}=f_{\varepsilon}, \\
(\alpha+\varepsilon) p_{\varepsilon}+\operatorname{div} u_{\varepsilon}=0,\left.\quad u_{\varepsilon}\right|_{\partial \Omega}=0 .
\end{gathered}
$$

For $\alpha=$ const it was proved that

$$
\left\|u_{\varepsilon}-u\right\|_{W_{2}^{1}}+\left\|p-p_{\varepsilon}\right\| \leq c \varepsilon .
$$

Below we will establish the validity of this estimate for the case of variable $\alpha$.

Note that $0 \leq \alpha \leq 1$. If $p \in L_{2}$ then by $p^{\prime}$ we will denote the orthogonal projection of $p$ onto the subspace of functions from the space $L_{2}$ which are orthogonal to unity (we will denote this subspace by $L_{2} / R_{1}$ ). Therefore, we have

$$
p=s+p^{\prime}, \quad s=\text { const }, \quad\left(p^{\prime}, 1\right) \equiv \int_{\Omega} p^{\prime} d x=0 .
$$

Let us prove the uniform boundedness of the functions $u_{\varepsilon}$ and $p_{\varepsilon}$. To this end, we take the scalar product of the first equation (3) by $u_{\varepsilon}$ and the scalar product of the second equation (3) by $p_{\varepsilon}$ and sum up the results; thus we obtain

$$
\left\|u_{\varepsilon}\right\|_{1}^{2}+\left((\alpha+\varepsilon) p_{\varepsilon}, p_{\varepsilon}\right)=\left(f_{\varepsilon}, u_{\varepsilon}\right) \text {. }
$$

From the first equation (3) for an arbitrary nonzero vector-function $\phi$ we obtain

$$
\frac{\left|\left(\nabla p_{\varepsilon}, \varphi\right)\right|}{\|\phi\|_{1}} \leq \frac{\left|\left(\nabla u_{\varepsilon}, \nabla \varphi\right)\right|}{\|\phi\|_{1}}+\frac{\left|\left(f_{\varepsilon}, \varphi\right)\right|}{\|\phi\|_{1}} \leq\left\|u_{\varepsilon}\right\|_{1}+\left\|f_{\varepsilon}\right\|_{-1} .
$$

We use the Babuška-Brezzi inequality from [4]:

$$
\|q\|_{L_{2}} \leq c_{0} \sup _{\varphi \in W_{2}^{\circ}} \frac{|(q, \operatorname{div} \varphi)|}{\|\varphi\|_{1}} .
$$

This inequality and the estimate (6) yield

$$
\left\|p_{\varepsilon}^{\prime}\right\| \leq c_{0}\left(\left\|u_{\varepsilon}\right\|_{1}+\left\|f_{\varepsilon}\right\|_{-1}\right) \text {. }
$$

Here and above we use the standard notations

$$
\|q\|=\|q\|_{L_{2}}, \quad\|f\|_{-1}=\sup _{\varphi \in W_{2}^{1}} \frac{(\varphi, f)}{\|\varphi\|_{1}}, \quad\|\varphi\|_{1}=\|\nabla \varphi\| .
$$

Since $\alpha+\varepsilon \geq \varepsilon>0$, the equality (5) gives the estimate

$$
\left\|u_{\varepsilon}\right\|_{1} \leq\left\|f_{\varepsilon}\right\|_{-1} \text {. }
$$

From this estimate and the inequality (7) we obtain

$$
\left\|u_{\varepsilon}\right\|_{1}+\left\|p_{\varepsilon}^{\prime}\right\| \leq\left(2 c_{0}+1\right)\left\|f_{\varepsilon}\right\|_{-1} .
$$

Finally, since $f_{\varepsilon} \rightarrow f$ the inequality (8) implies the existence of a constant $c_{1}$ independent of $\varepsilon$ such that the following inequality is satisfied:

$$
\left\|u_{\varepsilon}\right\|_{1}+\left\|p_{\varepsilon}^{\prime}\right\| \leq c_{1}\|f\|_{-1} .
$$

This means that the norms $\left\|u_{\varepsilon}\right\|_{1}$ and $\left\|p_{\varepsilon}^{\prime}\right\|$ are uniformly bounded with respect to $\varepsilon$. 
Let $p_{\varepsilon}=s_{\varepsilon}+p_{\varepsilon}^{\prime}$. Let us prove the uniform boundedness of $\left|s_{\varepsilon}\right|$. Using the expansion of $p_{\varepsilon}$ and estimating the right-hand side of the equality (5) by the $\varepsilon$-inequality we obtain

$$
\frac{3}{4}\left\|u_{\varepsilon}\right\|_{1}^{2}+s_{\varepsilon}^{2}(\alpha+\varepsilon, 1)+2 s_{\varepsilon}\left((\alpha+\varepsilon), p_{\varepsilon}^{\prime}\right)+\left((\alpha+\varepsilon) p_{\varepsilon}^{\prime}, p_{\varepsilon}^{\prime}\right) \leq c_{1}^{2}\left\|f_{\varepsilon}\right\|_{-1}^{2} .
$$

Therefore, from the estimates (9) and (10) we have

$$
\frac{3}{4}\left\|u_{\varepsilon}\right\|_{1}^{2}+s_{\varepsilon}^{2}(\alpha+\varepsilon, 1)+2 s_{\varepsilon}\left(\alpha+\varepsilon, p_{\varepsilon}^{\prime}\right)+\left((\alpha+\varepsilon) p_{\varepsilon}^{\prime}, p_{\varepsilon}^{\prime}\right)+\left\|p_{\varepsilon}^{\prime}\right\|^{2} \leq 2 c_{1}^{2}\|f\|_{-1}^{2} .
$$

Using the $\varepsilon$-inequality we can estimate the term $2 s_{\varepsilon}\left(\alpha+\varepsilon, p_{\varepsilon}^{\prime}\right)$ on the left-hand side of (11) as follows:

$$
\begin{aligned}
2\left|s_{\varepsilon}\left(\alpha+\varepsilon, p_{\varepsilon}^{\prime}\right)\right| & =2\left|\left(s_{\varepsilon} \sqrt{\alpha+\varepsilon}, \sqrt{\alpha+\varepsilon} p_{\varepsilon}^{\prime}\right)\right| \\
& \leq \varepsilon_{1} s_{\varepsilon}^{2}(\alpha+\varepsilon, 1)+\frac{1}{\varepsilon_{1}}\left((\alpha+\varepsilon) p_{\varepsilon}^{\prime}, p_{\varepsilon}^{\prime}\right) .
\end{aligned}
$$

Let $\varepsilon_{1} \leq 1$; substituting this inequality into the estimate (11) we obtain

(12) $\frac{3}{4}\left\|u_{\varepsilon}\right\|_{1}^{2}+\left(1-\varepsilon_{1}\right) s_{\varepsilon}^{2}(\alpha, 1)+\left(1-\frac{1}{\varepsilon_{1}}\right)\left((\alpha+\varepsilon) p_{\varepsilon}^{\prime}, p_{\varepsilon}^{\prime}\right)+\left\|p_{\varepsilon}^{\prime}\right\|^{2} \leq 2 c_{1}^{2}\|f\|_{-1}^{2}$.

Since we are mainly interested in the asymptotic behaviour as $\varepsilon \rightarrow 0$, we assume that $\varepsilon \leq 1$. Taking into account that $\alpha \leq 1$ and choosing $\varepsilon_{1}=4 / 5$ we obtain from the inequality (12) the estimate

$$
\frac{1}{5} s_{\varepsilon}^{2}(\alpha, 1)+\frac{1}{2}\left\|p_{\varepsilon}^{\prime}\right\|^{2} \leq c\|f\|_{-1}^{2} .
$$

But the inequality (9) yields the boundedness of the norm $p_{\varepsilon}^{\prime}$ and thus

$$
\left|s_{\varepsilon}\right| \leq \frac{c}{\sqrt{(\alpha, 1)}}\|f\|_{-1} .
$$

This gives the estimate of the form

$$
\left\|p_{\varepsilon}\right\|^{2}=s_{\varepsilon}^{2}+\left\|p_{\varepsilon}^{\prime}\right\|^{2} \leq c_{2}\|f\|_{-1}^{2} .
$$

Now we pass to the proof of the estimate (4). Let $q_{\varepsilon}=p-p_{\varepsilon}, v_{\varepsilon}=u-u_{\varepsilon}$. The errors $v_{\varepsilon}$ and $q_{\varepsilon}$ are found by solving the problem

$$
\begin{gathered}
-\Delta v_{\varepsilon}+\nabla q_{\varepsilon}=f-f_{\varepsilon}, \\
\alpha q_{\varepsilon}+\operatorname{div} v_{\varepsilon}=\varepsilon p_{\varepsilon},\left.\quad v_{\varepsilon}\right|_{\partial \Omega}=0 .
\end{gathered}
$$

Let us form the scalar product of the first equation (14) and $v_{\varepsilon}$ and the scalar product of the second equation (14) and $q_{\varepsilon}$. Summing up the results we obtain the equality

$$
\left\|v_{\varepsilon}\right\|_{1}^{2}+\left(\alpha q_{\varepsilon}, q_{\varepsilon}\right)=\left(f-f_{\varepsilon}, v_{\varepsilon}\right)+\varepsilon\left(p_{\varepsilon}, q_{\varepsilon}\right) .
$$

From the first equation (14) we obtain as above the following estimate:

$$
\left\|q_{\varepsilon}^{\prime}\right\| \leq c_{0}\left(\left\|v_{\varepsilon}\right\|_{1}+\left\|f-f_{\varepsilon}\right\|_{-1}\right) \text {. }
$$

Let us multiply both sides of the last inequality by an arbitrary constant $\lambda$ and take squares. Summing up this result and the equality (15) and estimating the 
right-hand side, we have

$$
\begin{aligned}
& \left(\frac{1}{2}-c_{0}^{2} \lambda\right)\left\|v_{\varepsilon}\right\|_{1}^{2}+\left(\alpha q_{\varepsilon}, q_{\varepsilon}\right)+\lambda\left\|q_{\varepsilon}^{\prime}\right\|^{2} \\
& \quad \leq \varepsilon \delta\left\|q_{\varepsilon}\right\|^{2}+\frac{\varepsilon}{4 \delta}\left\|p_{\varepsilon}\right\|^{2}+\left(\frac{1}{2}-c_{0}^{2} \lambda_{0}\right)\left\|f-f_{\varepsilon}\right\|_{-1}^{2} .
\end{aligned}
$$

Let $q_{\varepsilon}=l_{\varepsilon}+q_{\varepsilon}^{\prime}$ and $l_{\varepsilon}=$ const. Then the following sequence of relations holds:

$$
\begin{aligned}
\left(\alpha q_{\varepsilon}, q_{\varepsilon}\right)+\lambda\left\|q_{\varepsilon}^{\prime}\right\|^{2} & =l_{\varepsilon}^{2}(\alpha, 1)+2 l_{\varepsilon}\left(\alpha, q_{\varepsilon}^{\prime}\right)+\left(\alpha q_{\varepsilon}^{\prime}, q_{\varepsilon}^{\prime}\right)+\lambda\left\|q_{\varepsilon}^{\prime}\right\|^{2} \\
& \geq l_{\varepsilon}^{2}(\alpha, 1)+\alpha\left(q_{\varepsilon}^{\prime}, q_{\varepsilon}^{\prime}\right)+\left(\lambda q_{\varepsilon}^{\prime}, q_{\varepsilon}^{\prime}\right)-\varepsilon_{1} l_{\varepsilon_{1}}^{2}(\alpha, 1)+\frac{1}{\varepsilon_{1}}\left(\alpha q_{\varepsilon}^{\prime}, q_{\varepsilon}^{\prime}\right) \\
& \geq\left(1-\varepsilon_{1}\right) l_{\varepsilon}^{2}(\alpha, 1)+\left(\lambda+1-\frac{1}{\varepsilon_{1}}\right)\left\|q_{\varepsilon}^{\prime}\right\|^{2} .
\end{aligned}
$$

Let $\varepsilon_{1}=2 /(2+\lambda)$; then the last inequality yields the estimate

$$
\left(\alpha q_{\varepsilon}, q_{\varepsilon}\right)+\lambda\left\|q_{\varepsilon}^{\prime}\right\|^{2} \geq \frac{\lambda(\alpha, 1)}{2+\lambda} l_{\varepsilon}^{2}+\frac{\lambda}{2}\left\|q_{\varepsilon}^{\prime}\right\|^{2} \geq c_{3}\left\|q_{\varepsilon}\right\|^{2}
$$

where

$$
c_{3}=c_{3}(\lambda)=\min \left\{\frac{\lambda(\alpha, 1)}{2+\lambda}, \frac{\lambda}{2}\right\} .
$$

Note that $c>0$ since $(\alpha, 1)>0$ by assumption. Choose $\lambda=1 /\left(8 c_{0}^{2}\right)$ and $\delta=c_{3} /(2 \varepsilon)$. Then from the inequality (16) we obtain the estimate

$$
\frac{1}{4}\left\|v_{\varepsilon}\right\|_{1}^{2}+\frac{c_{3}}{2}\left\|q_{\varepsilon}\right\|^{2} \leq \frac{\varepsilon^{2}}{2 c_{3}}\left\|p_{\varepsilon}\right\|^{2}+c_{4}\left\|f-f_{\varepsilon}\right\|_{-1}^{2} .
$$

This estimate implies the convergences $v_{\varepsilon} \rightarrow 0$ and $q_{\varepsilon} \rightarrow 0$ as $\varepsilon \rightarrow 0$. Moreover, if the $f_{\varepsilon}$ converge to $f$ so that the estimate $\left\|f_{\varepsilon}-f\right\|_{-1} \leq c \varepsilon$ is satisfied then the convergence will be of first order in $\varepsilon$. Thus the estimate (4) is true.

So we have proved the following theorem:

THEOREM 1. Let the domain $\Omega$, the functions $f$ and $f_{\varepsilon}$ and the coefficient $\alpha$ be such that the generalised solutions of the problems (2) and (3) exist and are unique and $f_{\varepsilon}-f \rightarrow 0$. Then the solution $\left(u_{\varepsilon}, p_{\varepsilon}\right)$ of the problem (3) converges to $(u, p)$ with $\varepsilon \rightarrow 0$ where $(u, p)$ is the solution of the problem (2). Moreover, if $\left\|f_{\varepsilon}-f\right\|_{-1} \leq c \varepsilon$ then we can estimate the rate of convergence of $\left(u_{\varepsilon}, p_{\varepsilon}\right)$ to $(u, p)$. Namely, in this case

$$
\left\|u_{\varepsilon}-u\right\|_{1}+\left\|p_{\varepsilon}-p\right\| \leq c \varepsilon
$$

Remark 1 . The convergence of $\left(u_{\varepsilon}, p_{\varepsilon}\right)$ to $(u, p)$ holds true not only in the continuous case but also in the discrete case when the boundary value problems (2) and (3) are approximated by a finite element method or by a finite difference one. Here it is necessary that the discrete analogue of the BabuškaBrezzi inequality is valid while the operators $\nabla$ and div are approximated so that $\left(\nabla^{h}\right)^{*}=-\operatorname{div}^{h}$. 


\section{References}

[1] R. Temam, Navier-Stokes Equations, North-Holland, 1979.

[2] G. M. Kobel'kov, On equivalent norms of subspaces of $L_{2}$, Anal. Math. 3 (1977) (in Russian).

[3] —, On an iterative method for solution of the difference equations of elasticity theory, Dokl. Akad. Nauk SSSR 233 (5) (1977) (in Russian).

[4] G. M. Kobel'kov and V. D. Valedinskiŭ, On the inequality $\|p\|_{L_{2}} \leq c\|\nabla p\|_{-1}$ and its finite dimensional image, Soviet J. Numer. Anal. Math. Modelling 1 (3) (1986). 